ESAIM: PROCEEDINGS AND SURVEYS, September 2014, Vol. 45, p. 359-368

J.-S. Dhersin, Editor

\title{
DÉTECTION DE COURANTS MARINS CÔTIERS À PARTIR DE SÉQUENCES VIDÉO*
}

\author{
Stanislas Larnier $^{1}$, Rafael Almar ${ }^{2}$, Rodrigo Cienfuegos ${ }^{3}$ And Antoine Lejay ${ }^{1}$
}

\begin{abstract}
In nearshore applications, the estimation of currents is of primary importance since it controls the sediment transport and coastal evolution. Direct estimation of currents using in-situ instruments is difficult and costly. Low cost remote sensing systems based on video observations constitute a promising alternative when the drifting sea foam left after the passage of breaking waves is visible. Only few methods have been proposed in the literature to take advantage of this.

In this paper we describe two new methods: one based on the full video frames and one based on longshore timestacks that necessitates less data. The first approach is an extension of Particle Image Velocimetry method. The second approach uses the Radon transform applied on the time series derived from timestacks to produce an estimate of the longshore component of nearshore surface currents detected from the foam signature in video images.

The videos used in this article come from a field campaign conducted in the Mataquito rivermouth area in the Maule region (Chile). They are part of a study on the post-tsunami coastal recovery of the Mataquito area after 2010 Chilean Tsunami. Field tests comparison was also made against in situ current meter from the 2008 Truc Vert experiment in Aquitaine (France). It took place during a tempest which has provoked a sandbar evolution.

Résumé. Les courants marins à proximité d'une côte contrôlent le transport de sédiment et son évolution. Or leur estimation par des instruments sur site est difficile et coûteuse. Les systèmes de télédétection reposant sur des observations constituent une alternative prometteuse lorsque la mousse dérivante laissée après le passage de vagues déferlantes est visible. Pourtant, seules quelques méthodes ont été proposées à ce jour dans la littérature.

Dans cet article, nous décrivons deux nouvelles méthodes : l'une basée sur les images vidéos considérées dans leur globalité et l'autre basée sur des timestacks le long de la côte. La première approche est une extension de la méthode Particle Image Velocimetry (PIV). La seconde approche produit une estimation de la composante parallèle à la côte des courants de surface en utilisant la transformée de Radon sur des séries temporelles de données en une dimension. Cette dernière nécessite moins de données.

Les vidéos utilisées dans cet article proviennent d'une campagne menée sur la zone de l'embouchure de Mataquito dans la région de Maule au Chili. Elles font partie d'une étude sur l'évolution côtière de la zone de Mataquito après le tsunami chilien de 2010. Une comparaison a été également réalisée avec les mesures d'un courantomètre prises lors de la campagne du Truc Vert de 2008 en Aquitaine, France. Elle a eu lieu lors d'une tempête qui a provoqué l'évolution d'une barre sableuse.
\end{abstract}

\footnotetext{
* Nous remercions la région Lorraine, l'équipe associée franco-chilienne Anestoc-Tosca d'Inria, et le Centre Inria CIRIC au Chili pour leurs financements.

1 Université de Lorraine, Institut Elie Cartan de Lorraine, UMR 7502, Vandoeuvre-lès-Nancy, F-54506, France - CNRS, Institut Elie Cartan de Lorraine, UMR 7502, Vandoeuvre-lès-Nancy, F-54506, France — Inria, Villers-lès-Nancy, F-54600, France. stanislas.larnier@inria.fr, antoine.lejay@inria.fr

2 Institut de Recherche pour le Développement (IRD) / LEGOS, Toulouse, France. rafael.almar@ird.fr

3 Departamento de Ingeniería Hidráulica y Ambiental, Pontificia Universidad Católica, Santiago, Chile. racienfu@ing.puc.cl
}

(C) EDP Sciences, SMAI 2014 


\section{INTRODUCTION}

Les mouvements des vagues de houle peuvent être utilisés pour engendrer de l'électricité. On parle alors d'énergie houlomotrice ou d'énergie des vagues. Elle reste peu utilisée bien qu'il y ait 15 à 20 fois plus d'énergie par mètre carré que pour l'énergie solaire ou éolienne tout en étant plus régulière [11]. La recherche dans ce domaine est en plein développement. Disposant d'une longue côte où l'Océan Pacifique est fortement énergétique, le Chili, pays partenaire du projet, envisage d'investir dans cette énergie renouvelable.

Le projet franco-chilien multi-disciplinaire à l'origine de cette étude souhaite utiliser les données vidéo, dispositif à bas coût, pour déterminer le potentiel énergétique d'un site. Pour cela, il faut estimer la période moyenne, la célérité moyenne, et la hauteur moyenne des vagues, la vitesse moyenne des courants côtiers, l'angle moyen du front de vagues par rapport à la côte ainsi que la bathymétrie (topographie du fond sous-marin).

Les techniques de vidéos côtières se développent depuis plus d'une vingtaine d'années. Elles ont été employées pour détecter la forme des bandes sableuses submergées [9], la période et la direction des vagues [10], la localisation de la ligne de côte [13] et la bathymétrie proche de la côte [14]. Comparée à l'emploi de radars ou de capteurs, cette technique a l'avantage d'avoir un faible coût d'installation et d'utilisation [1]. Déterminer correctement les variables géophysiques nécessite d'être à même de transformer les coordonnées de l'image en coordonnées réelles. Une telle transformation se détermine à partir de points de contrôle de coordonnées géophysiques connues [7].

Dans cet article, on s'intéresse uniquement à l'estimation de la vitesse moyenne $V$ des courants côtiers en utilisant le déplacement des amas de mousse créés lorsqu'une vague se brise. Les trajectoires suivies par ces amas de mousse dépendent du courant et des vagues. Des méthodes estiment uniquement la composante des courants parallèles à la côte [3]. D'autres utilisent toute la vidéo comme les Particle Image Velocimetry (PIV) [6].

Nous avons travaillé sur des données issues de deux campagnes. La campagne du Chili a été menée à l'embouchure du fleuve Mataquito dans la région de Maule. Ces vidéos font partie d'une étude sur la reconstruction naturelle côtière dans la région de Mataquito après le tsunami chilien de 2010 [15]. À la différence de l'installation classique d'autres campagnes, où les caméras sont sur un mât proche de la plage, le système est placé sur une montagne de la cordillère côtière.
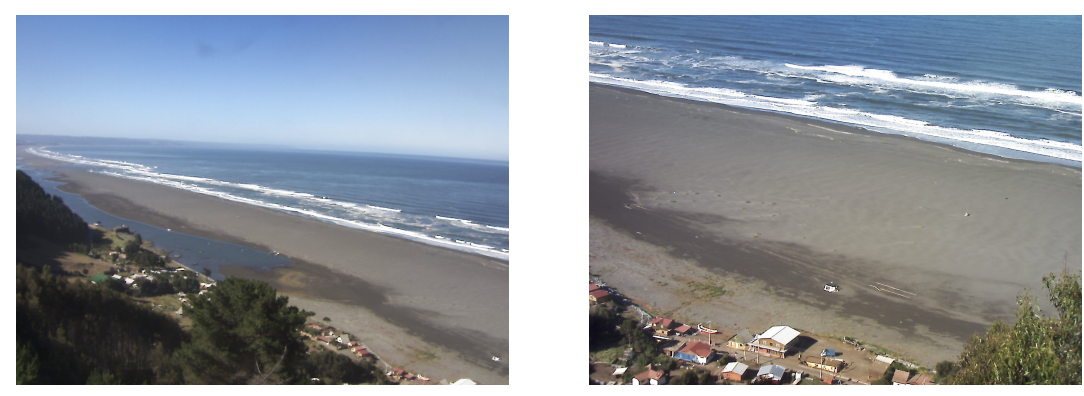

Figure 1. Vues des caméras de Mataquito.

Les vidéos de la campagne du Truc Vert ont été prises lors d'une expérimentation durant la tempête de 2008 qui a frappé la région Aquitaine en France. Cette étude fait partie du programme ECORS. Elle a permis de noter l'évolution d'une barre sableuse due à cet événement énergétique [12].

La Section 1 aborde une amélioration de la technique PIV pour les images vidéos complètes. La Section 2 présente des pré-traitements envisageables pour séparer les vagues et les résidus de mousse ce qui en facilite l'étude, et également le problème des indésirables (oiseaux, nageurs, promeneurs, bateaux, ...). Dans la Section 3, une nouvelle approche basée sur la transformée de Radon est présentée pour traiter les timestacks parallèles à la côte. 

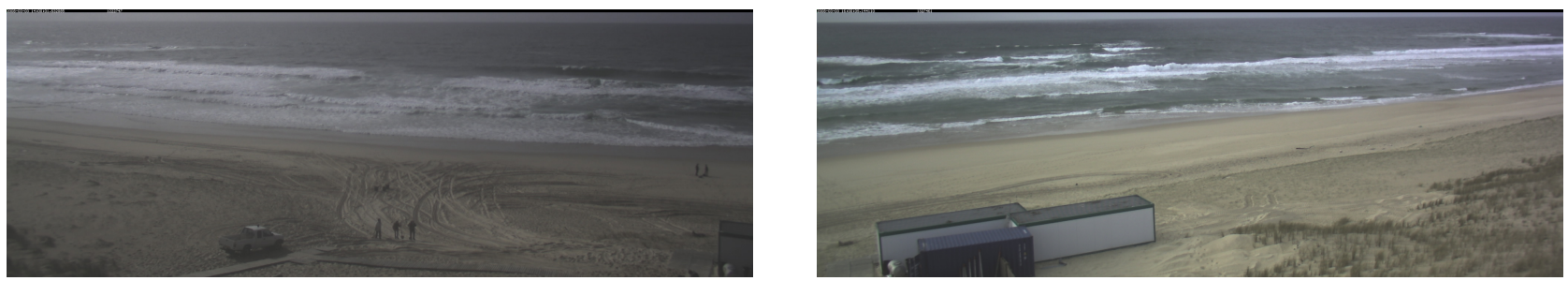

Figure 2. Vues des caméras du Truc Vert.

\section{Estimation des COURANTS À PARTiR DE la CORRÉlation ENTRE LES imageS}

\subsection{Principe de la Particle Image Velocimetry}
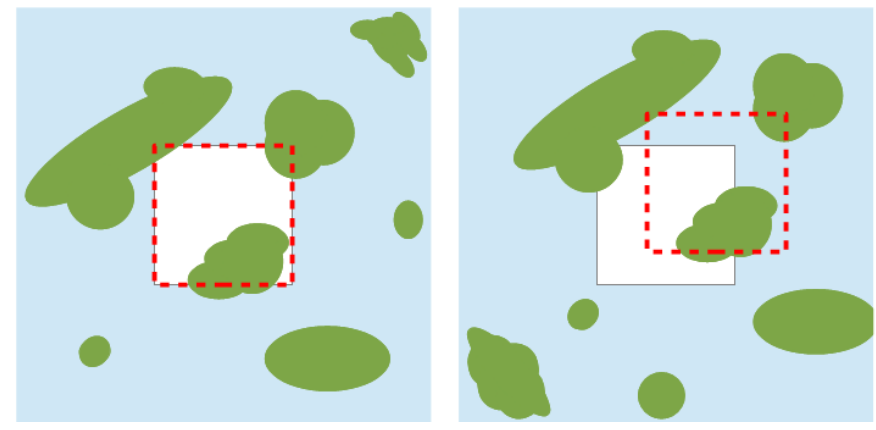

Figure 3. Schéma d'explication pour la Particle Image Velocimetry

Une image vidéo est découpée en portions selon une grille. Dans la méthode PIV, le déplacement d'une portion est estimé en cherchant les portions d'images à l'instant suivant qui sont les plus corrélées avec cette première. C'est une technique de block-matching utilisant la corrélation comme critère métrique de sélection. La Figure 3 illustre cette approche. Le carré blanc est notre portion d'intérêt. À droite, la portion entourée de rouge est la plus corrélée avec celle d'origine. La distance parcourue est donnée par la distance entre les centres de gravité de ces deux portions. Cette technique fonctionne correctement en bassin pour des colorants. En pratique, pour obtenir les courants sur un intervalle de temps à partir de vidéos côtières, il faut moyenner les déplacements obtenus en supprimant les plus aberrants et ensuite tenir compte de l'effet de déplacement dû au front de vagues vers la côte.

L'approche que nous proposons est d'employer la PIV uniquement sur des sous-images comprises entre des fronts de vagues. En plus de la recherche des portions d'images corrélées, la méthode cherche à minimiser des fonctions coûts pour obtenir un déplacement proche en terme d'orientation et d'amplitude du déplacement des sous-images voisines.

Dans cet article, nous ne considérons que le cas d'un océan agité qui engendre beaucoup de mousse et donc d'informations contrairement à l'océan paisible avec peu d'amas de mousse qui dérivent au gré des courants. Dans ce deuxième cas, nous conseillons de suivre et classifier les objets en mouvement (fronts de vagues, brisures, amas de mousse) en s'intéressant à des informations liées aux déplacements, plutôt que des informations colorimétriques qui sont sujettes à des variations dues à la météo et à la luminosité. Proches de la côte, les vagues se déplacent quasiment perpendiculairement à la côte ce qui n'est en règle générale pas le cas des résidus de mousse. 


\subsection{Amélioration de la Particle Image Velocimetry}

En traitement d'image, la corrélation croisée sert à mesurer la similitude entre deux images. Soient $\mathcal{Z}$ un ensemble de pixels formant une portion carrée de l'image et $(u, v)$ un couple d'entier relatifs. En pratique, si l'on s'intéresse à la distance entre deux portions $(f(x, y))_{(x, y) \in \mathcal{Z}}$ et $(g(x-u, y-v))_{(x, y) \in \mathcal{Z}}$ d'images $f$ et $g$, il est plus avantageux d'utiliser la corrélation croisée normalisée $[5,8]$ définie par

$$
\gamma_{f, g}(u, v)=\frac{\sum_{(x, y) \in \mathcal{Z}}(f(x, y)-\bar{f})(g(x-u, y-v)-\bar{g})}{\sqrt{\sum_{(x, y) \in \mathcal{Z}}(f(x, y)-\bar{f})^{2} \cdot \sum_{(x, y) \in \mathcal{Z}}(g(x-u, y-v)-\bar{g})^{2}}},
$$

où $\bar{f}$ et $\bar{g}$ sont les moyennes de $(f(x, y))_{(x, y) \in \mathcal{Z}}$ et $(g(x-u, y-v))_{(x, y) \in \mathcal{Z}}$.

L'image est découpée en blocs de même taille. L'algorithme cherche la meilleure corrélation croisée normalisée entre un bloc et tous les blocs possibles de l'image suivante compris dans un voisinage de son centre. La Figure 5 présente les deux images de la même zone à deux temps proches. Le champ de vecteurs en bas à gauche est construit en ne considérant que le maximum de la corrélation croisée normalisée. Le calcul n'est pas effectué pour les blocs qui contiennent un bâton, l'algorithme renvoie une interpolation avec les valeurs des blocs voisins.

On constate que dans certaines zones, les vecteurs ont des longueurs et orientations proches et crédibles. Ce n'est cependant pas le cas partout. Pour améliorer ces résultats, on ajoute des contraintes par le biais de trois énergies qui prennent respectivement en compte la corrélation, l'orientation et la longueur des vecteurs voisins :

$$
\begin{gathered}
J_{\text {corr }}\left(I_{1}, I_{2}, \Theta, R\right)=\sum_{i=1}^{M} \gamma_{I_{1}, I_{2}}(\phi(\Theta(i), R(i))), \quad J_{\text {angl }}(\Theta)=\sum_{i=1}^{M} \frac{1}{|\mathcal{V}(i)|} \sum_{j \in \mathcal{V}(i)} \cos (\Theta(j)-\Theta(i)) \\
\text { et } J_{\text {long }}(R)=\sum_{i=1}^{M} \frac{1}{|\mathcal{V}(i)|} \sum_{j \in \mathcal{V}(i)}(R(j)-R(i))^{2} .
\end{gathered}
$$

Les images sont notées $I_{1}$ et $I_{2}$. Les variables $\Theta$ et $R$ contiennent respectivement les orientations et les longueurs des vecteurs des $M$ blocs de l'image. La notation $\mathcal{V}(i)$ correspond au voisinage du bloc $i$ qui contient 8 voisins (sauf pour les blocs frontières). La fonction $\phi$ est une transformation des coordonnées polaires en coordonnées cartésiennes. Dans la suite du texte les énergies locales pour un bloc $i$ seront les mêmes, de sorte que la somme sur les $M$ blocs peut être ignorée.

Le champ de vecteurs sera donc modifié de façon à maximiser $J_{\text {corr }}$ et $J_{\text {angl }}$ et à minimiser $J_{\text {long }}$ par une procédure itérative, inspirée du modèle d'Ising, dont une étape est illustrée par la Figure 4.

Un nouveau champ proche du précédent est tiré aléatoirement. Il est conservé s'il donne une énergie locale plus faible qu'avec la situation précédente. Afin que l'algorithme ne soit pas bloqué dans un minimum local, des idées utilisées en optimisation stochastique et simulation en mécanique statistique sont employées. Des situations qui peuvent être moins bonnes sont acceptées dans la limite de certains critères.
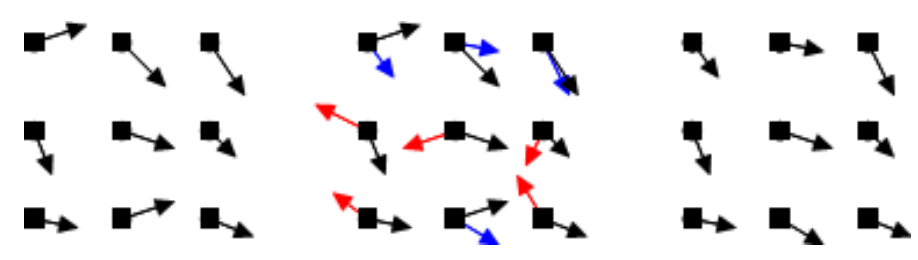

Figure 4. Schéma d'explication pour la recherche du meilleur champ 


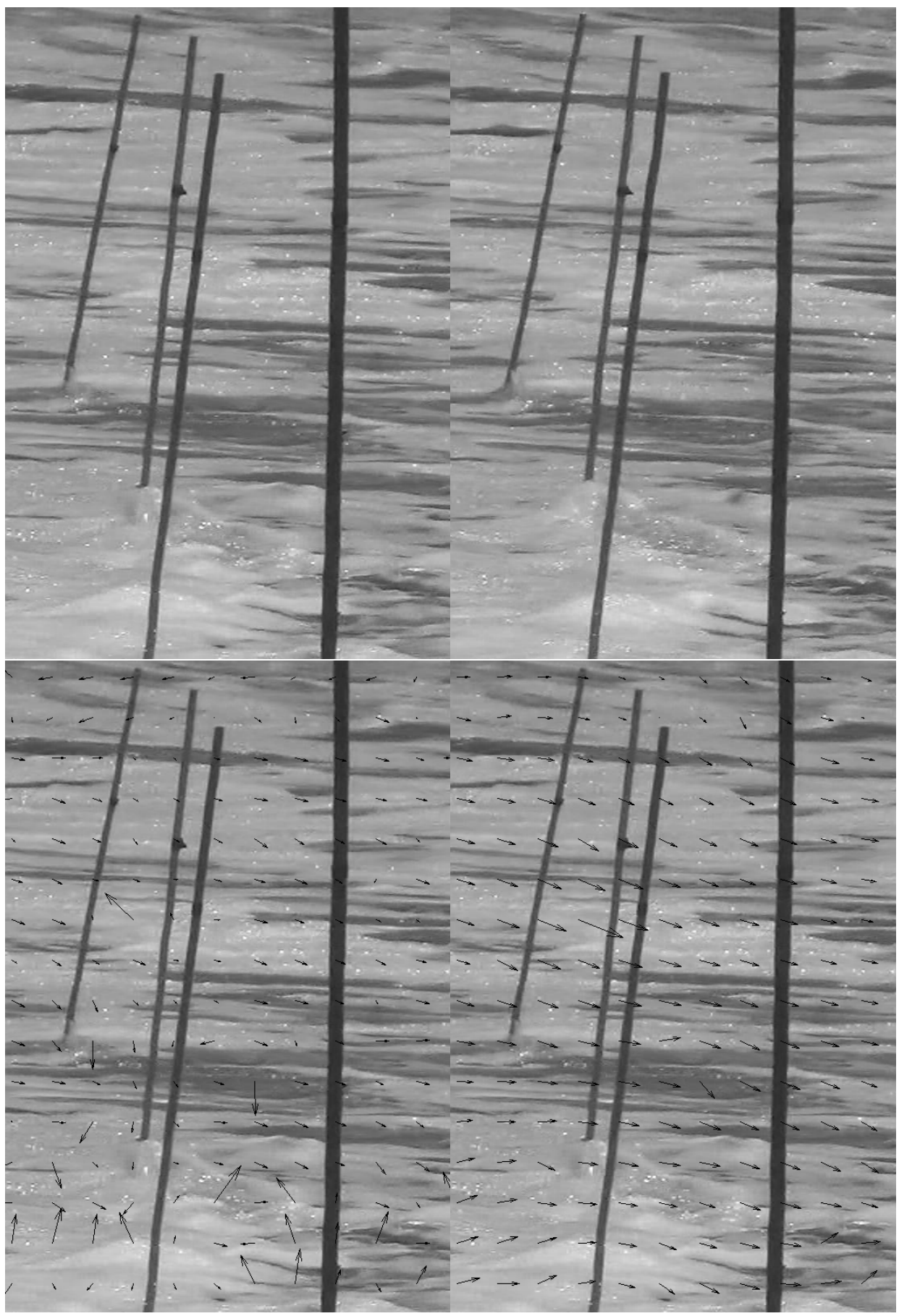

FIGURE 5. En haut, de gauche à droite, image au temps $t$ et image au temps $t+2$. En bas, de gauche à droite, champs de déplacement en considérant uniquement la corrélation puis avec les trois énergies. La vidéo a été acquise à partir d'une caméra sur trépied au Chili. 
La Figure 4 permet de mieux appréhender le concept. L'image de gauche présente le champ de vecteurs initial. On tire aléatoirement d'autres vecteurs présentés en bleu et rouge dans la seconde image. Les bleus font baisser les énergies locales du point de vue de l'orientation et du rayon. On les conserve si les résultats de corrélations sont bons. Les rouges ne conviennent pas et on conserve alors les mêmes vecteurs que dans le champ initial. La troisième image est le résultat de ces opérations. Le processus est réitéré pour obtenir un champ convenable. Pour converger vers une solution, on diminue progressivement les variations d'angles et de rayons.

L'algorithme est le suivant. Il prend en entrée $N$, le nombre d'itérations.

(1) Initialisation du champ de vecteurs en maximisant $J_{\text {corr }}\left(I_{1}, I_{2}, \Theta, R\right), n=1$

(2) À l'itération $n$, pour chaque bloc $i$, tirages aléatoires avec loi uniforme d'un angle $\delta \theta_{i}$ dans $\left[-\frac{\pi}{n}, \frac{\pi}{n}\right]$, d'un coefficient $a_{i}$ entre $\left[\frac{n}{N}, 2-\frac{n}{N}\right]$.

(3) Pour chaque bloc $i$, calcul des six énergies locales suivantes $J_{\text {corr }}^{i}\left(I_{1}, I_{2}, \theta_{i}+\delta \theta_{i}, a_{i} r_{i}\right), J_{\text {angl }}^{i}\left(\theta_{i}+\delta \theta_{i}\right)$, $J_{\text {long }}^{i}\left(a_{i} r_{i}\right), J_{\text {corr }}^{i}\left(I_{1}, I_{2}, \theta_{i}, r_{i}\right), J_{\text {angl }}^{i}\left(\theta_{i}\right)$ et $J_{\text {long }}^{i}\left(r_{i}\right)$.

(4) Pour chaque bloc $i$,

(a) Si $J_{\text {angl }}^{i}\left(\theta_{i}+\delta \theta_{i}\right) \geq J_{\text {angl }}^{i}\left(\theta_{i}\right)$ alors poursuivre à l'étape (b),

(b) Si $\frac{90}{100} J_{\text {long }}^{i}\left(a_{i} r_{i}\right) \leq J_{\text {long }}^{i}\left(r_{i}\right)$ alors poursuivre à l'étape $(\mathrm{c})$,

(c) Si $J_{\text {corr }}^{i}\left(I_{1}, I_{2}, \theta_{i}+\delta \theta_{i}, a_{i} r_{i}\right) \geq U J_{\text {corr }}^{i}\left(I_{1}, I_{2}, \theta_{i}, r_{i}\right)$ où $U$ est un tirage aléatoire uniforme sur [0,1], alors $\theta_{i}=\theta_{i}+\delta \theta_{i}$ et $r_{i}=a_{i} r_{i}$.

(5) Tant que $n<N, n=n+1$, retourner à l'étape 2 .

La Figure 5 montre l'application de cette méthode : le champ de vecteur initial (en bas à gauche) est obtenu en maximisant les corrélations. Le champ de vecteurs en bas à droite est obtenu par application de cet algorithme.

\section{Pré-traitements}

\subsection{Séparation vagues et résidus de mousse}

Pour l'expérience proche de la mer, avec les bâtons, présentée dans la Figure 5, la caméra est sur trépied et les vagues correspondent à de fortes zones de mouvement horizontal. Un seuil colorimétrique peut suffire en sélectionnant les lignes horizontales qui sont les plus blanches, soit en détectant les lignes horizontales qui varient le plus. Dans le cadre plus général des vidéos qui nous intéresse, une technique basée sur la transformée de Fourier permet de détecter les crêtes de vagues [16]. Nous proposons de séparer les vagues et les résidus de mousse en utilisant la transformée de Radon.

La transformée de Radon [4] d'une fonction de deux variables $f$ est donnée par l'intégrale double selon une direction $\theta$ et un rayon $\rho$ :

$$
R(\rho, \theta)=\int_{-\infty}^{\infty} \int_{-\infty}^{\infty} f(x, y) \delta(x \cos (\theta)+y \sin (\theta)-\rho) \mathrm{d} x \mathrm{~d} y .
$$

Ici, $\theta$ et $\rho$ sont respectivement l'angle et le rayon qui définissent la ligne, en coordonnées polaires, sur laquelle la fonction est projetée. Les variables $x$ et $y$ représentent les coordonnées orthonormées et $\delta$ est la fonction de Dirac. Ainsi, $f$ est intégrée le long de la ligne définie par $\rho=x \cos (\theta)+y \sin (\theta)$.

La transformée de Radon est plus particulièrement employée dans le traitement d'images médicales, notamment en tomographie. Dans le cas des vidéos côtières, la transformée de Radon a été employée sur des timestacks perpendiculaires à la côte afin de séparer les vagues incidentes de celles réfléchies [2].

La Figure 6 présente deux résultats de séparation de vagues et de résidus de mousse en employant la transformée de Radon. Pour chacun des résultats, le maximum du sinogramme résultant de la transformée de Radon donne une estimation de l'angle $\varphi$ représentant l'orientation du front de vagues. La première image filtrée conserve uniquement les informations entre les angles $\varphi-5^{\circ}$ et $\varphi+5^{\circ}$. La deuxième image filtrée correspond au reste de l'information. Il est envisageable de travailler sur ces images pour connaître la localisation des vagues et ne pratiquer la PIV améliorée ou le suivi de résidus de mousse qu'entre deux crêtes. 


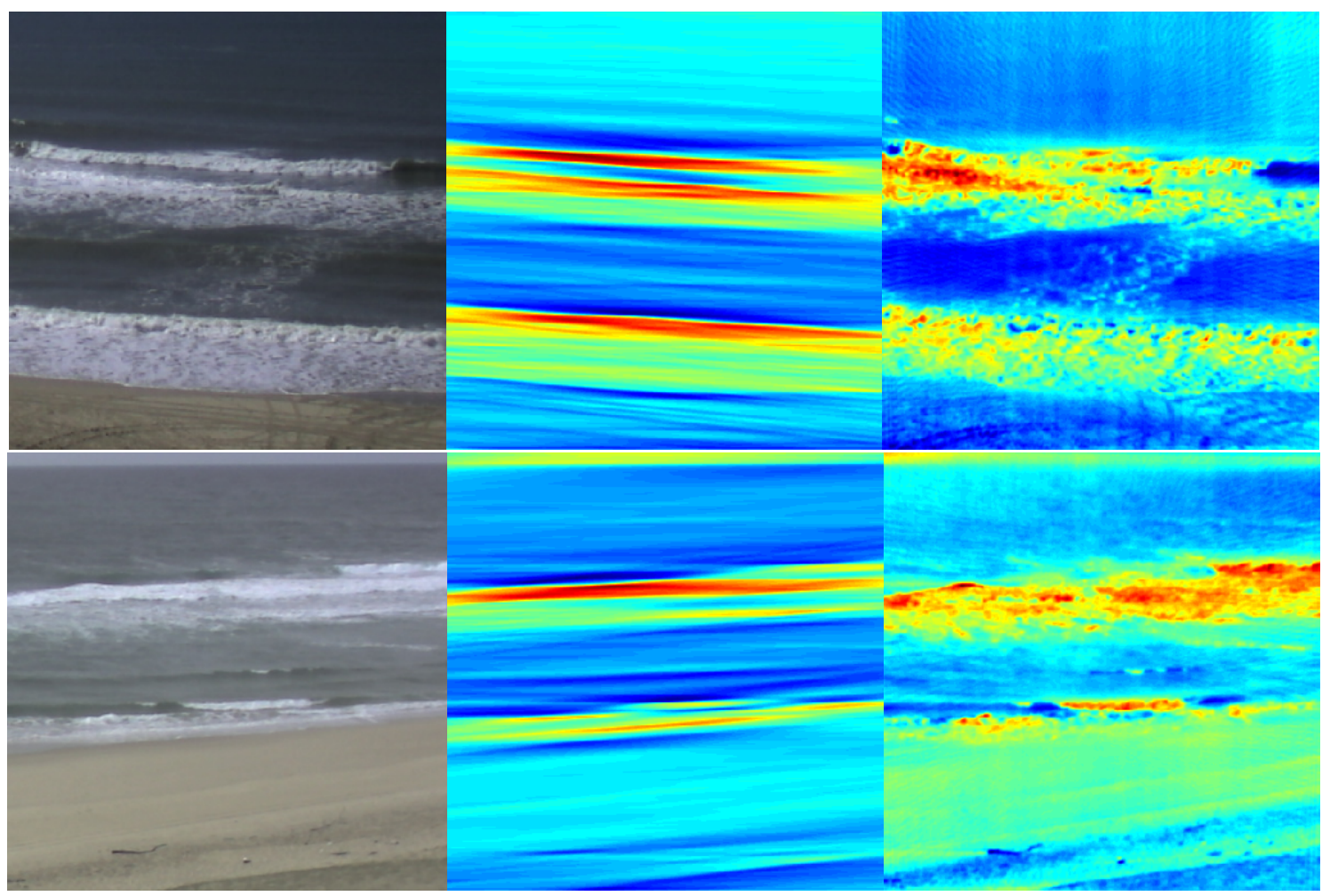

FIgURE 6. De gauche à droite, images d'origine, filtrées pour faire ressortir les vagues, filtrées pour faire ressortir la mousse.

\subsection{Détection et suivi des indésirables}

Des éléments en mouvement autres que les vagues et les résidus de mousse peuvent perturber les algorithmes. Nous appellerons indésirables de tels éléments, par exemple les piétons, les nageurs, les oiseaux ou encore les bateaux.

La Figure 7 présente un vautour sur le site de Mataquito. Cet oiseau présente un fort contraste avec le reste de l'image. Comme tout autre élément de couleur très différente de ce que l'on s'attend à trouver dans l'image, il est facile à détecter et à suivre par une simple analyse de colorimétrie et des techniques classiques de suivi d'objets (Voir Figure 7).
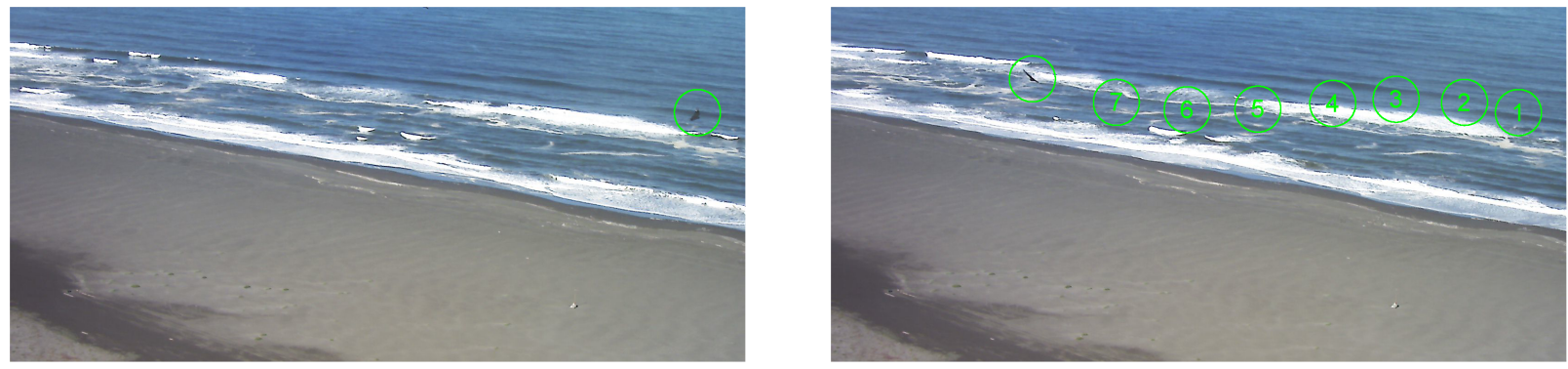

Figure 7. De gauche à droite, détection et suivi d'un vautour sur le site de Mataquito. 
Il n'en va pas de même pour d'autres éléments indésirables qui sont proches d'un point de vue colorimétrique $\mathrm{du}$ fond et qui apparaissent dès leurs entrées dans le champ de la caméra au niveau de zones d'intérêt. Citons le cas des mouettes et des pélicans.

\section{Estimation Des COURANTs À PARTiR DE TIMESTACKS}

\subsection{Timestacks parallèles à la côte}

Les timestacks sont des images créées à partir d'une séquence temporelle d'une ligne de pixels choisie. Chaque ligne horizontale représente la capture vidéo d'une ligne traversant le champ de la caméra à un instant donné, telle que la ligne rouge, parallèle à la côte, de la Figure 8. Chaque ligne verticale donne l'évolution temporelle d'un point fixé sur cette ligne. La Figure 9 présente le timestack résultant correspondant à environ quatre périodes du front de vague à partir de la ligne rouge présentée à la Figure 8. Les fronts de vagues donnent les traits horizontaux blancs. Les résidus de mousse qui dérivent forment des traits obliques. Leurs orientations permettent de déterminer la vitesse des courants de surface. Sur cette image, le timestack a été agrandi du point de vue temporel pour avoir une meilleure vision des phénomènes.

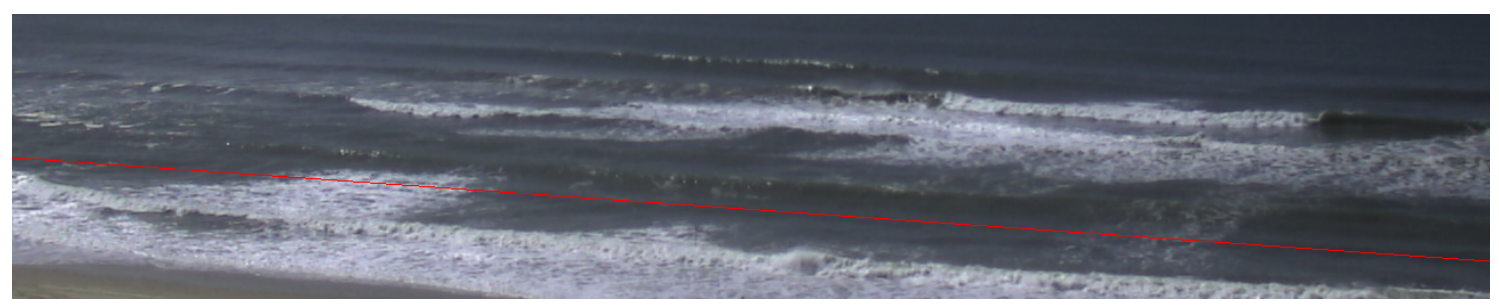

Figure 8. Image du site du Truc Vert, la ligne rouge représente la position du timestack parallèle au front de vague

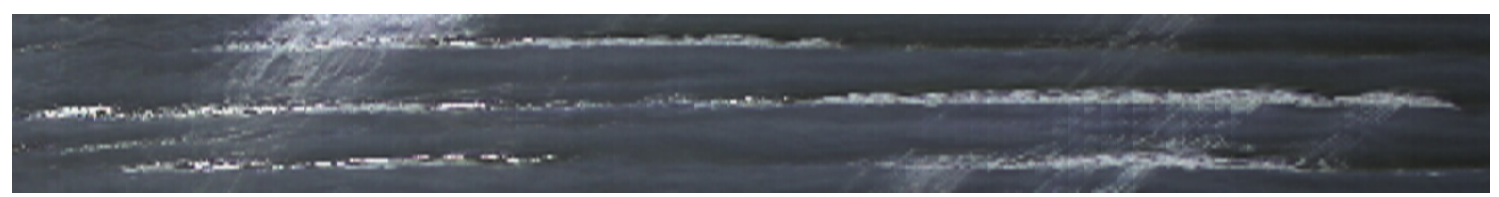

FiguRE 9. Timestack associé : les lignes horizontales sont les crêtes des vagues, l'orientation des autres lignes formées par les résidus des mousses donne la vitesse des courants de surface le long de la côte.

\subsection{Approche basée sur la transformée de Radon}

L'algorithme est le suivant :

(1) Filtrage de Radon du timestack afin de supprimer les lignes horizontales (vagues) et verticales (artefacts capteur caméra) avec une variation de plus ou moins $8^{\circ}$;

(2) Filtrage temporel avec un filtre passe haut;

(3) Filtrage spatial pour ne garder que l'information haute-fréquence ;

(4) Étude des coefficients de Radon pour déterminer les maxima locaux ;

(5) Suppression des valeurs extrêmes en dehors des valeurs limites ;

(6) Moyennisation des coefficients pour déterminer la meilleure orientation. 

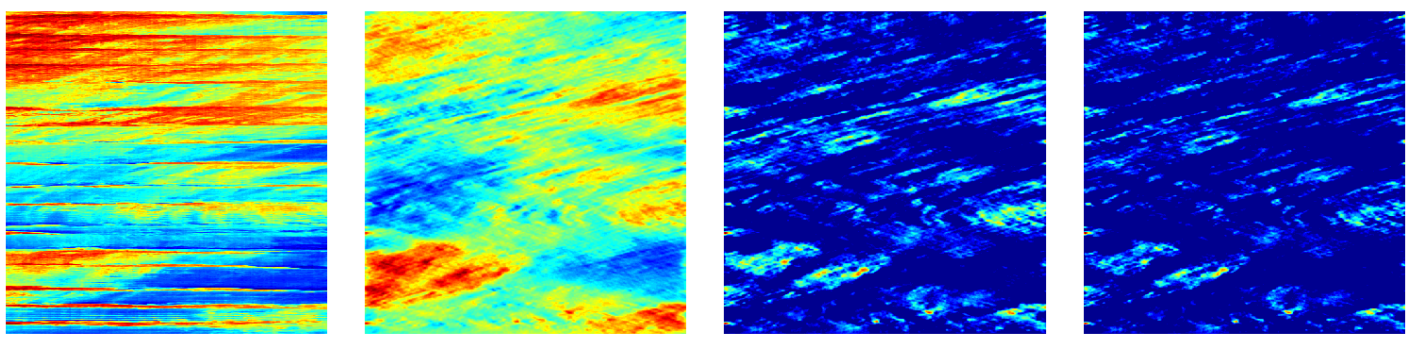

Figure 10. De gauche à droite, image d'origine, filtrée avec Radon, puis filtrée en temporel, puis filtrée en spatial

Les figures suivantes illustrent les étapes de l'algorithme sur la journée du 10 mars de 7 heures 45 à 8 heures pour le site du Truc Vert. La Figure 10 présente le timestack et les étapes successives des filtres sur l'image. L'image de gauche dans la Figure 11 présente l'image filtrée sur laquelle l'orientation du courant va être déterminée. Bien que quelques zones n'aient pas la bonne orientation, l'image obtenue est majoritairement correcte.

Les valeurs de Radon pour l'image filtrée sont données dans l'image de droite de la Figure 11 (l'image d'origine a été tournée d'un quart de tour) avec les maxima locaux au-dessus d'un certain seuil entourés d'un cercle rouge. Le capteur a mesuré une vitesse moyenne de $-0.64 \mathrm{~m} . \mathrm{s}^{-1}$ et l'algorithme renvoie $-0.66 \mathrm{~m} . \mathrm{s}^{-1}$.
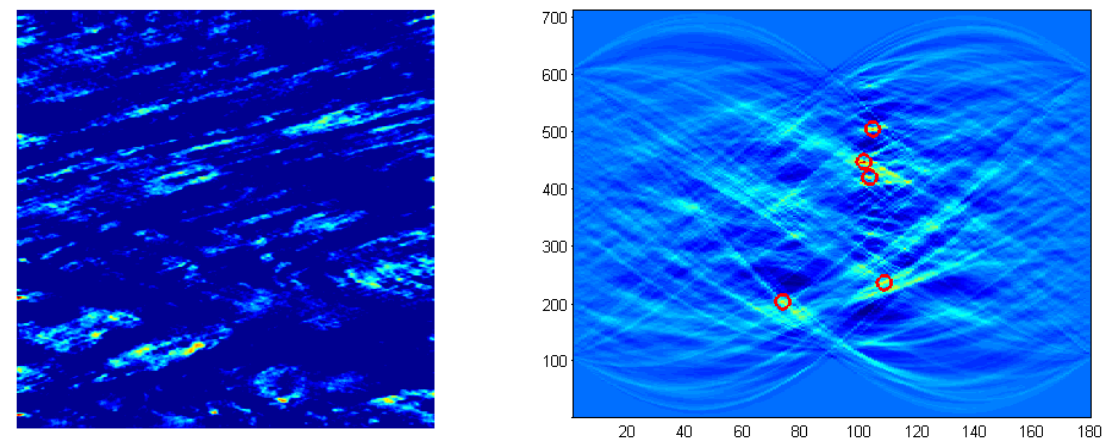

FiguRE 11. De gauche à droite, image filtrée et la transformée de Radon de cette image avec les maxima locaux élevés entourés d'un cercle rouge.

La Figure 12 présente deux timestacks pris le long de la côte sur le site de Mataquito à des positions identiques mais dans des conditions de luminosité différentes. Un opérateur humain a trouvé respectivement $13.8^{\circ}$ et $12.6^{\circ}$ pour le premier et le second timestack, contre $12.5^{\circ}$ et $13.6^{\circ}$ pour l'algorithme. L'algorithme donne des résultats similaires à ceux d'un opérateur humain.

\section{Conclusion}

Cet article présente deux méthodes pour estimer la vitesse moyenne des courants marins côtiers de surface à partir de données vidéo. La vitesse des courants affecte l'énergie houlomotrice. Elle est également un paramètre important pour étudier les transports de sédiment et l'évolution de la côte.

La première méthode proposée améliore la technique de Particule Image Velocimetry. Elle nécessite des prétraitements pour détecter ou séparer vagues et résidus de mousse, et supprimer tout indésirable. Des recherches sont encore à faire pour déterminer les meilleures approches pour ces pré-traitements. 

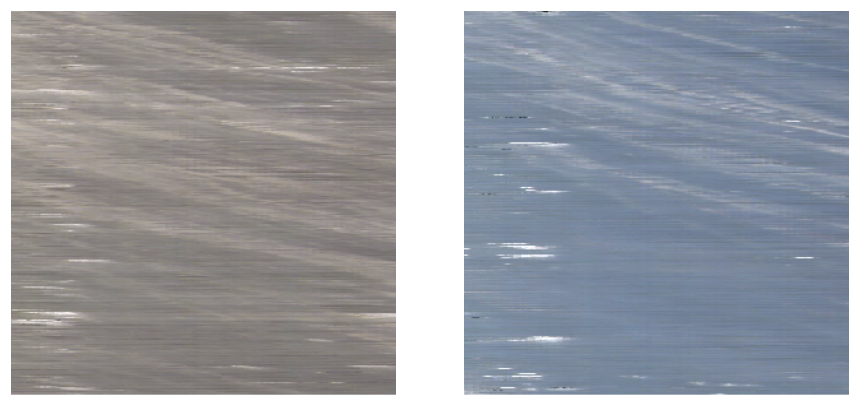

Figure 12. Deux timestacks avec des conditions de luminosité différentes sur le site de Mataquito.

La seconde approche est moins coûteuse en données et les premiers résultats sont encourageants. Les tests comparatifs avec des données terrains vont se poursuivre afin d'en connaître sa limitation. Une étude comparative avec des méthodes existantes [3] est également envisagée.

D'autres pistes de recherches qui peuvent être développées sont l'analyse de la qualité de la scène, l'adaptation à des conditions extrêmes (brouillard, pluie) et la détection d'indésirables uniquement à partir des timestacks.

\section{REFERENCES}

[1] R. Almar. Morphodynamique littorale haute fréquence par imagerie vidéo. Thèse de doctorat, Université Bordeaux 1, France, 2009.

[2] R. Almar, P. Bonneton, H. Michallet, R. Cienfuegos, B.G. Ruessink, and M. Tissier. On the use of the Radon transform in studying nearshore wave dynamics. In Proceeding of Coastal Dynamics, 24-28 June 2013, Arcachon, France, pages 73-82, 2013.

[3] C. C. Chickadel, R. A. Holman, and M. H. Freilich. An optical technique for the measurement of longshore currents. J. Geophys. Research: Oceans, 108(C11), 2003.

[4] T. G. Feeman. The mathematics of medical imaging, A beginner's guide. Springer Undergraduate Texts in Mathematics and Technology. Springer, New York, 2010.

[5] R.M. Haralick and L.G. Shapiro. Computer and robot vision. Computer and Robot Vision. Addison-Wesley Pub. Co., 1993.

[6] K.T. Holland, J.A. Puleo, and T.N. Kooney. Quantification of swash flows using video-based particle image velocimetry. Coast. Eng., 44(2):65-77, 2001.

[7] R.A. Holman, A.H. Sallenger Jr., T.C. Lippman, and J.W. Haines. The application of video image processing to the study of nearshore processes. Oceanography, 6(3):78-85, 1993.

[8] J.P. Lewis. Fast normalized cross-correlation. Technical report, Industrial Light \& Magic, 1995.

[9] T. C. Lippmann and R. A. Holman. The spatial and temporal variability of sand bar morphology. J. Geophys. Research: Oceans, 95(C7):11575-11590, 1990.

[10] T.C. Lippmann and R.A. Holman. Phase speed and angle of breaking waves measured with video techniques. In N.C. Kraus, K.J. Gingerich, and D.L. Kriebel, editors, Coastal Sediments, pages 542-556, 1991.

[11] A. Muetze and J.G. Vining. Ocean wave energy conversion - a survey. In 41st IAS Annual Meeting. Conference Record of the 2006 IEEE Industry Applications Conference, volume 3, pages 1410-1417, 2006.

[12] S. Parisot, J.P. abd Capo, S. Bujan, J. Moreau, A. Réjas, V. Hanquiez, R. Almar, and al. Treatment of topographic and bathymetric data acquired at the Truc-Vert Beach (SW France) during the ECORS mission. Journal of Coastal Research, SI 56(2):1786-1790, 2009.

[13] N.G. Plant and R.A. Holman. Intertidal beach profile estimation using video images. Mar. Geol., 140:1-24, 1997.

[14] H. F. Stockdon and R. A. Holman. Estimation of wave phase speed and nearshore bathymetry from video imagery. J. Geophys. Research: Oceans, 105(C9):22015-22033, 2000.

[15] M. Villagran, R. Cienfuegos, P. Catalan, and R. Almar. Morphological response of central Chile sandy beaches to the 8.8 Mw 2010 earthquake and tsunami. In Proceeding of Coastal Dynamics, 24-28 June 2013, Arcachon, France, pages 1823-1834, 2013.

[16] J. Yoo. Nonlinear Bathymetry Inversion Based on Wave Property Estimation from Nearshore Video Imagery. Thèse de doctorat, Georgia Institute of Technology, 2007. 